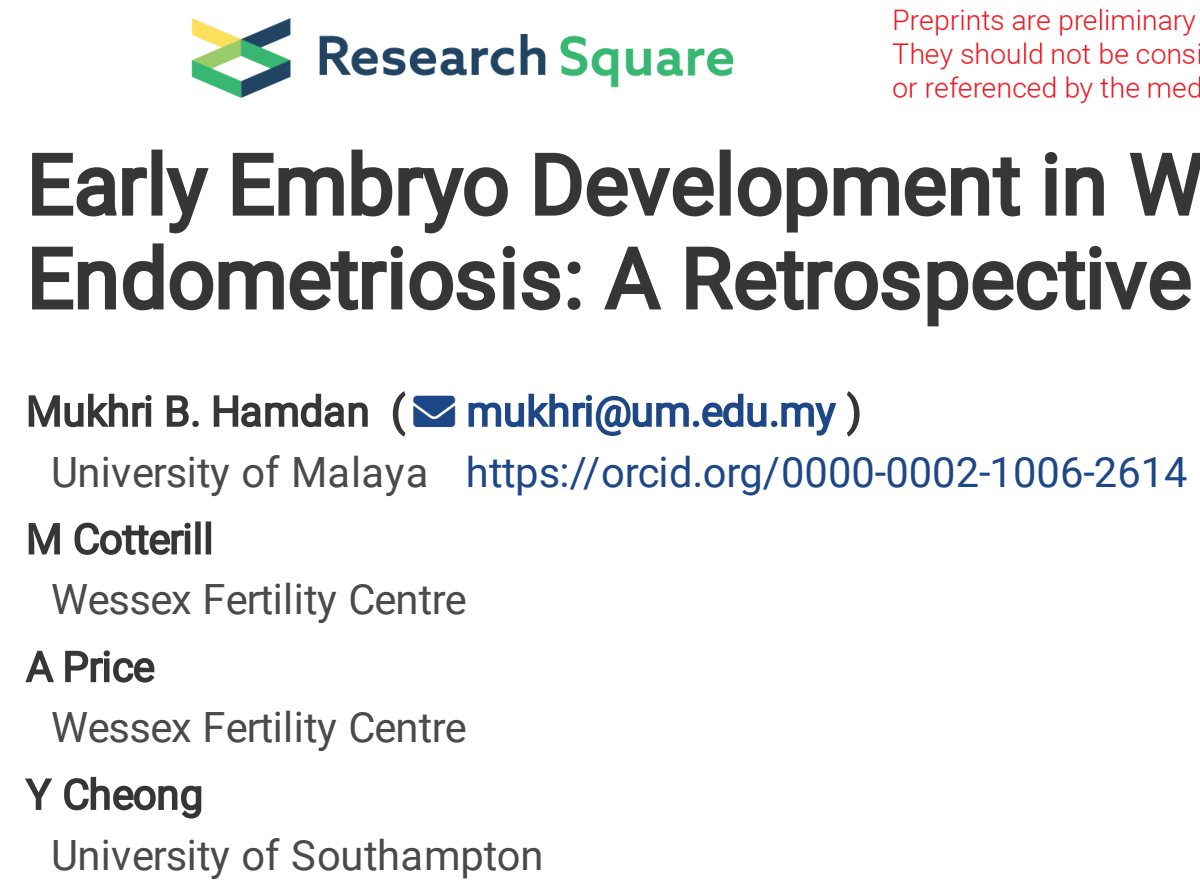

\author{
Mukhri B. Hamdan ( $\nabla$ mukhri@um.edu.my ) \\ University of Malaya https://orcid.org/0000-0002-1006-2614 \\ M Cotterill \\ Wessex Fertility Centre \\ A Price \\ Wessex Fertility Centre \\ $Y$ Cheong \\ University of Southampton
}

\title{
Early Embryo Development in Women with Endometriosis: A Retrospective Cohort Study
}

Research article

Keywords: Endometriosis, embryology, time-lapse, embryo decelopment

Posted Date: June 15th, 2020

DOI: https://doi.org/10.21203/rs.3.rs-33579/v1

License: (9) This work is licensed under a Creative Commons Attribution 4.0 International License. Read Full License 


\section{Abstract}

Background: Endometriosis is known to be detrimental to fertility in many ways. Evidence drawn from studies on oocyte recipient cycles suggests that the oocyte quality of women with endometriosis is compromised. Research that specifically examined the association between embryo quality and endometriosis/endometrioma are scarce and the question if endometriosis affects oocyte and/or embryo quality remain controversial. This study aims to evaluate embryo quality of women with endometriosis (EN) and without endometriosis (tubal factor [TF], unexplained infertility [UE]).

Methods: Multi-centre retrospective study was performed. Treatment cycles and embryology records of women with and without endometriosis undergoing IVF was reviewed via IDEAS ${ }^{\mathrm{TM}}$ database. Number of oocytes collected per mature follicle, fertilisation rate, pregnancy rate, morphology and morphometric assessment of embryo at all stages were evaluated

Results: Total of 678 women who had IVF treatment was analysed (EN, $n=89 ; T F, n=214 ; U E, n=375)$. The mean age for each group was EN, 34.4 $\pm 3.3 ; T F, 33.5 \pm 3.7$ and $34.6 \pm 3.2$ respectively. Number of mature follicles $(>14 \mathrm{~mm})$ on the day of trigger injection was similar $(P=0.75)$ between all groups. Percentage of oocytes collected per mature follicle was lower in endometriosis compared to other groups $(P=0.01 ; E N$, $65 \pm 23 ; T F, 76 \pm 20 ; U E, 71 \pm 24)$. Higher percentages of embryos fail to achieve 8-cell stage in EN compared to control groups $(P=0.02 ; E N, 4.0 \pm 1.6 T F, 1.2 \pm 0.4 ; U E, 1.5 \pm 0.4$. Percentages of embryos at all grades (Grade1-4) per women were similar between the comparison groups $(P>0.05)$. Endometriosis did not impair $(P>0.05)$ blastocyst development or the development of Fully Expanded Hatching Blastocysts (FEHB).

Conclusions: This study finds women with endometriosis have lower number of oocyte per total number of follicle. The presence of endometriosis is associated with a higher rate of early embryo arrest, which implicates poor oocyte quality. However endometriosis has no effect on the embryo quality beyond the 8cell-stage of embryo development

\section{Background}

A significant number of subfertile women with endometriosis may eventually require Artificial Reproductive Techniques (ART) with in-vitro fertilisation (IVF) and/or intra-cytoplasmic sperm injection (ICSI) to conceive. Up to $15 \%$ of women undergoing ART have endometriosis (Senapati et al., 2016). Endometriosis is known to be associated with subfertility although its pathophysiology is still unclear. Endometriosis can be detrimental to fertility at the level of the endometrium, oocyte and/ or embryo (Filippi et al., 2014, Reinblatt et al., 2011b).

Research from our group and others showed that women with endometriosis have a lower number of oocytes retrieved per cycle of ART (Hamdan et al., 2015b, Barnhart et al., 2002, Hamdan et al., 2015a, Harb et al., 2013, Lin et al., 2012), and those with endometrioma require a higher dose of FSH stimulation and have a higher cancellation rate during their IVF cycles (Hamdan et al., 2015a). Animal studies 
suggest that oocyte and embryo development were poorer when exposed to follicular and peritoneal fluid of women with endometriosis (Da Broi et al., 2014, Mansour et al., 2010, Hamdan et al., 2016). Studies on oocyte recipient cycles showed poorer reproductive outcome in recipients who received oocytes from women with endometriosis, thus, strongly suggesting that the oocyte quality of women with endometriosis is compromised (Diaz et al., 2000, Simon et al., 1994), although the vast majority of previous studies directly examining the impact of endometriosis oocyte and embryo quality and development fail to conclude that endometriosis detrimentally impact on oocyte and/or embryo quality as observed in ART cycles (Filippi et al., 2014, Reinblatt et al., 2011a, Dong et al., 2013, Al-Fadhli et al., 2006, Lin et al., 2012, Mekaru et al., 2013, Suzuki et al., 2005). The oocytes from women with endometriosis have structurally and functionally abnormal mitochondria (Xu et al., 2015) and a higher rate of aneuploidy (Gianaroli et al., 2010) compared to controls, although reports on the aberrant morphology of oocyte meiotic spindle are controversial(Mansour et al., 2009, Rajani et al., 2012) .

The current available studies examining the oocyte/embryo quality for women with and without endometriosis suffer from the following limitations. Embryo morphological assessment (Munne et al., 2007 ) is traditionally subjected to a high intra- and inter-observer variability when considering factors associated with morphological dysmorphisms such as fragmentation, asymmetry, and multinucleation despite the recent consensus on standardising embryo assessment(Alpha Scientists in Reproductive and Embryology, 2011). Only one study utilised a standardised objective embryo morphological assessment (Filippi et al., 2014, Alpha Scientists in Reproductive and Embryology, 2011) Most studies made their assessments on D3 rather than D5 embryo assessment and none of the studies utilised data obtained from time-lapse technology. The majority of the studies assessed the embryo quality as a secondary outcome rather than as their primary outcome.

This study aims to assess the oocyte and embryo development based on recently performed ART cycles, using standardised criteria for the assessment of embryo development, to evaluate the quality of oocytes and embryos in with endometriosis compared to those with no endometriosis.

- Methods

- Data extraction

This study was a retrospective case control study, performed in two tertiary referral fertility centres in the United Kingdom. Anonymised data from IDEAS V. $6^{\text {TM }}$ (Electronic IVF database, Mellowood Medical, USA) from January 2011 to December 2014 in both fertility centres were extracted, analysed, and reviewed. Data obtained from the database were exported to Excel (Microsoft, USA) before data analysis was performed using statistical analysis package.

- Study group and inclusion/exclusion criteria

This study included women under 40 years old who underwent IVF treatment using their own gametes. Oocyte donor cycles (altruistic or sharer) or couples with co-existing male factor subfertility that required intra-cytoplasmic sperm injection (ICSI) was excluded. 
Study group (Endometriosis, EN) consisted of women who were subfertile for at least 1 year with endometriosis, diagnosed laparoscopically and/or sonographically, irrespective of the disease severity and duration. All the women who had laparoscopically diagnosis and treatment of endometriosis were collectively included in the study group regardless of the type of surgical treatment performed.

Our control groups included women with tubal factor (TF) subfertility or unexplained subfertility (UE) defined as being subfertile for at least 1 year with no identified reasons for subfertility (normal hormonal profile, patent tube(s), and normozoospermia). Women were considered to be in the tubal factor group (TF) if they were subfertile for at least 1 year with evidence of tubal disease in either one or both tubes. If the tubes were blocked resulting in hydrosalphinges, the normal practice in the UK would be to remove or clip the tube(s). They do not have a history of endometriosis, with the absence of any current signs or symptoms of endometriosis, and have no ultrasound evidence of endometriosis.

Ovarian stimulation protocols were limited to the antagonist cycle and long agonist cycle. Short flare agonist cycles were excluded. There was no restriction on the type of stimulation drugs used; however recombinant FSH was the predominant drug of choice, with a starting dose based on the age and $\mathrm{AMH}$ level, in accordance to local dosing protocol. The day of trigger (HCG) was determined by the presence of 3 or more follicles over $17 \mathrm{~mm}$. The number of oocytes on the folliculogram $>14 \mathrm{~mm}$ was an arbitrary level of cut-off taken to compare the oocyte retrieval rates between the two groups from follicles that were most likely mature at the time of oocyte collection. This is of particular interest because work from our group and others have shown possibility of oocyte DNA damage as a sequelae of endometriosis which impacts on follicular maturation and development (Hamdan et al., 2016, Mansour et al., 2010).

- Oocytes and Embryos assessment

Qualified and trained embryologists from each centre performed the oocytes and embryo assessment. Following oocyte retrieval procedure, oocytes were incubated in the culture media until the in vitro fertilisation procedure. Where possible, the collected oocytes were scored according to the appearance of cumulus cells, zona pellucida, and cytoplasm (Alpha Scientists in Reproductive and Embryology, 2011).

Irrespective of the oocytes score, insemination was performed at approximately $1400 \mathrm{H}$ in the afternoon of the same day of egg collection. Fertilisation check was done $17 \pm 1$ hours post insemination (HPI). Normal fertilisation was defined as presence of 2 polar bodies with two centrally located pronuclei that are evenly sized.

At $68 \pm 1 \mathrm{HPI}$, all embryos were reassessed and scored using standardised scoring system(Alpha Scientists in Reproductive and Embryology, 2011) according to the cell number, fragmentation rate, and multinucleation. The scoring was documented in the embryology record electronically. At this stage, fertilised oocytes that have not cleaved were categorised as arrested at D1 and were discarded.

At D3, selected women underwent embryo transfer procedure, which was decided according to the respective unit's embryo transfer criteria and protocol. Embryos that were not transferred at D3 were 
cultured to blastocyst stage. At D5 of culture, embryos were rescored. Suitable good quality blastocysts were usually frozen on the same day or the day after (D6) if the criteria were met.

\section{Time-lapse embryo assessment}

As an alternative to conventional daily time-specific embryo morphological check, Eeva ${ }^{\mathrm{TM}}$ was used for embryo monitoring following fertilisation check. Eeva ${ }^{\mathrm{TM}}$ uses non-invasive, time-lapse imaging of embryos during undisturbed incubation, which detects critical and subtle differences in cell division. The timelapse data was available only in one of the two centres.

- Outcome measures definitions

Number of oocytes retrieved is defined as the total number of oocytes collected per oocyte collection procedure. Percentage of oocytes per mature follicles is defined as the total number of oocytes collected over total number of follicles (measuring $>14 \mathrm{~mm}$ on the day of trigger). The number of fertilised oocytes is defined as the number of normally fertilised oocytes per number of patient. Fertilisation rate is defined as the number of normally fertilised oocytes per number of inseminated oocytes. D1 arrested embryo is defined as fertilised oocytes in which development has arrested and has not reached cleavage stage. At Day 3, individual embryo was scored into 4 Grades (1, 2, 3, and 4). Grade 1 and Grade 2 embryos at day three were grouped and deemed as poor quality oocytes, whereas Grade 3 and Grade 4 were grouped and deemed as good quality oocytes. Number of embryo transferred and cryopreserved are define as number of embryo transferred into the uterus and frozen respectively, over number of patients. Utilisation rate is defined as the percentage of embryos transferred or cryopreserved, over the number of oocytes retrieved. Blastulation rate is defined as the percentage of blastocysts over the number of fertilised oocytes. Fully expanded and hatching blastocyst (FEHB) is defined as good quality blastocysts at Grade 4 and above. Clinical pregnancy is defined as the presence of at least one fetal heart. For time-lapse data, the time taken for the fertilised embryos to develop from 2-pronuclei stage to 2 cell (P2) and 3 cells (P3) were recorded.

- Statistical analysis and data representation

Statistical analysis was performed using unpaired t-test for parametric data. Fischer exact test or chi square test was used for categorical data. A p-value $<0.05$ was considered to be significant. One-way ANOVA was used for comparison of more than 2 groups with the normally distributed data. If the ANOVA showed statistically significant difference, post hoc analysis was done using Tukey's post-hoc test. Kruskal-Wallis test was used for comparison of more than 2 groups with non-parametric data. All statistical analyses were performed using the Statistical Package for Social Sciences software, version 18 (SPSS Inc., Chicago, IL, USA) or Prism6 (GraphPad, USA) except effect size, which was calculated using the Cohen $d$ Test (G*Power, USA). Data is expressed as mean \pm standard deviation (SD), or standard error (SE) or as percentage \pm SD or SE. 


\section{Results}

\section{Demographic characteristics of the patients between groups}

A total of 678 women who had IVF treatment were analysed and they comprised of 3 groups (EN, $n=89$; $T F, n=214 ; U E, n=375$, Table 1). The mean age (years $\pm S D$ ) for each group was $E N, 34.4 \pm 3.3, T F, 33.5 \pm$ 3.7, and UE, $34.6 \pm 3.2$ (Table 1). Data for age was normally distributed and there was no difference found between EN with the other groups (TF, $P=0.17$; UE, $P=0.78$, Table 1). Women in TF group were, however, younger than UE groups $(\mathrm{P}=0.002)$, with a small effect size, $d=0.3214$ (Cohen $d$ Test).

On the other hand, AMH levels were not normally distributed and therefore non-parametric test was performed which showed no significant difference between the three groups $[P=0.432$; Median: EN, 11.7 (6.16 to 20.15); TF, 15.7 (7.27 to 24.3); UE, 12.28 (6.70 to 22.93), (Table 1)]. There were significantly more women in endometriosis group $(n=37,42 \%)$ who had long protocol compared to the control groups (TF, $n=17,8 \%, P=0.0001 ; U E, n=15,4 \%, P=0.0001$ ).

\section{Women with endometriosis have lower number of oocytes retrieved per follicle $>14 \mathrm{~mm}$}

The mean number of follicles ( $>14 \mathrm{~mm}$ ) on the day of trigger injection was similar between all groups ( $P$ $=0.75 ; \mathrm{EN}, 12 \pm 5 ; \mathrm{TF}, 13 \pm 6 ; \mathrm{UE}, 13 \pm 6$; Table 1 ). The mean number of oocytes (mean $\pm \mathrm{S}$.E.) collected at oocyte retrieval was similar $(P=0.31 ; E N, 8.6 \pm 0.5 ; T F, 9.5 \pm 0.4 ; U E, 9.2 \pm 0.2$; Table 1$)$. The mean percentage of oocytes (mean \pm S.E.) collected per follicle $>14 \mathrm{~mm}$ was significantly lower in endometriosis compared to tubal factor groups ( $P=0.01 ; E N, 65 \pm 3.4$ and $T F, 76 \pm 1.7$; Table 2), but similar between endometriosis and unexplained group $(P=0.34 ; E N, 65 \pm 3.4$ and $\mathrm{UE}, 70 \pm 1.5$; Table 1).

\section{Women with endometriosis have a similar fertilisation rate compared to controls}

All the collected oocytes were inseminated irrespective of their maturity, and the mean number of fertilised oocytes (Mean $\pm S E$ ) per woman was found to be similar between groups ( $P=0.31$, EN, $5.7 \pm 0.4$; TF, $6.4 \pm 0.3 ; \mathrm{UE}, 6.3 \pm 0.2$; Table 2). The percentages of fertilised oocytes were calculated over the number of oocytes inseminated to represent the actual fertilisation rate. There was no difference in the mean percentage (Mean $\pm \mathrm{SE}$ ) of oocytes fertilised per number of oocytes inseminated, across the three groups, $(P=0.76, E N, 66 \pm 3.3 ; T F, 76 \pm 1.7 ; \mathrm{UE}, 71 \pm 1.5 ;$ Table 1).

At this stage, unfertilised oocytes were discarded, and fertilised oocytes were incubated and cultured until another routine embryo assessment at D3. The number and the rate of fertilised embryo that have successfully cleaved were examined. 
Women with endometriosis have a higher early embryo arrest and poorer progression to 8-cell cleavage rate

Following the fertilisation check, fertilised oocytes were cultured and incubated before reassessed at $68 \pm$ 1 hours post insemination. During the assessment, fertilised oocytes were expected to cleave and progress to 8-cell stage. At this stage of the assessment, the majority of the oocytes were found to have developed further, but some were arrested and failed to cleave. The mean number (Mean \pm SE) of arrested embryo per woman was similar amongst the three groups $(P=0.688 ; E N, 0.14 \pm 0.4 ; T F, 0.08 \pm 0.3 ; U E$, $0.11 \pm 0.7$; Table 1). The rate of embryo arrested per fertilised oocyte (percentage Mean $\pm S E$ ) was significantly lower in women with endometriosis compared to controls $(P=0.02$; $E N, 4.0 \pm 1.6 T F, 1.2 \pm 0.4$; $\mathrm{UE}, 1.5 \pm 0.4$; Table 1). Post hoc analysis showed a higher percentage of arrested embryos in the EN compared to the TF groups $[P=0.02 ; \operatorname{MD} 2.77,95 \% \mathrm{Cl}(0.33,5.21)$; Table 1$]$ and UE groups $[P=0.03, M D$ $2.51,95 \% \mathrm{Cl}(0.22,4.79)$; Table 1].

\section{Embryos development and quality at D3 were unaffected by endometriosis}

Embryo assessment at D3 was usually performed to monitor the development of individual embryos and to enable the decision for choosing suitable embryo/s for transfer or for extended culture until blastocyst stage, with the clinical circumstances taken into account. In this study, the mean percentages of embryos +/- SE amongst all grades of embryo per women were similar between the comparison groups; Grade 1 embryos ( $P=0.651$; $E N, 23 \pm 3.4 ; T F, 20 \pm 1.9 ; \mathrm{UE}, 22 \pm 1.5$; Table 2$)$, Grade 2 embryos $(P=0.242 ; E N, 42 \pm$ 3.2; TF, $43 \pm 2.0$; $U E, 39 \pm 1.5$; Table 2), Grade 3 embryos ( $P=0.768 ; E N, 24 \pm 2.8 ; T F, 26 \pm 1.8 ; U E, 26 \pm 1.3$; Table 2), and Grade 4 embryos ( $P=0.607 ; E N, 12 \pm 2.4 ; T F, 12 \pm 2.2 ; \mathrm{UE}, 13 \pm 2.2 ;$ Table 2$)$.

Considering the embryo assessment tool was subjective and have high inter-and intra-observer variability, embryos graded as 1 and 2 were pooled as 'good quality' embryos, whilst embryos graded as 3 and 4 pooled as 'poor quality'.

There was no difference of percentages across the three comparison groups whether the comparison was made with good quality embryos ( $P=0.59$; $E N, 64 \pm 4.0 ; T F, 63 \pm 2.3 ; U E, 61 \pm 1.8$ ) or the poor quality embryos $(P=0.59 ; E N, 36 \pm 4.0 ; T F, 37 \pm 2.3 ; U E, 39 \pm 1.8)$.

Following day 3 assessments, these embryos were either selected to be transferred or continue to be cultured to D5, and some may be of sufficient quality to be suitable for freezing. Next, the number and quality of the embryos that progressed to blastocyst was examined.

The development of blastocyst or Fully Expanded Hatching Blastocysts (FEHB) were similar in women with endometriosis compared with controls

Women who had blastocyst transferred at D5 or D6 were accounted for in the blastocyst number and blastulation rate. Those who already had embryo transferred before D5 were excluded in this analysis. 
The number of embryos (mean +/- S.D.) at blastocyst stage per woman were similar amongst all the three groups $(P=0.62 ; E N, 4.2 \pm 2.5 ; T F, 3.8 \pm 2.2 ; U E, 3.7 \pm 2.2 ;$ Table 2$)$ and the blastulation rate were also similar across all groups ( $P=0.50 ; E N, 51 \pm 4.3 ; T F, 49 \pm 2.3 ; \mathrm{UE}, 46 \pm 1.9 ;$ Table 2$)$.

FEHB per women were found to be similar amongst the three groups $(P=0.38 ; E N, 2.8 \pm 0.4 ; T F, 3.0 \pm 0.2$; UE, $2.6 \pm 0.2$; Table 2), and FEHB rate per fertilised oocytes was not statistically different across the three groups ( $P=0.34$; EN, $35 \pm 3.9$ TF, $38 \pm 2.4$; UE, $34 \pm 2.2$; Table 2).

\section{Utilisation of embryos was similar between women with and without endometriosis}

Utilisation rate represented the number of embryos transferred and number of embryos frozen per cycle individually and also in combination. The utilisation rate was the percentage of total transferred and frozen embryos per woman over the total number of oocytes retrieved per woman.

The number of embryos transferred per cycle (mean +/- S.D.) was the same among the comparison groups $(P=0.18 ; E N, 1.5 \pm 0.7 ; T F, 1.5 \pm 0.6 ; U E, 1.5 \pm 0.6 ; 3)$. The mean number of embryos cryopreserved per cycle (Mean $\pm \mathrm{SE}$ ) was also the same across all groups $(\mathrm{P}=0.86 ; \mathrm{EN}, 1.6 \pm 2.3 ; \mathrm{TF}, 1.5 \pm 2.3 ; \mathrm{UE}, 1.6 \pm$ 2.3; Table 3).

The number of embryos utilised were similar among the comparison groups $(P=0.53 ; E N, 2.8 \pm 0.2 ; T F$, $2.9 \pm 0.1$; UE, $3.0 \pm 0.1$; Table 3 ). The percentages of utilisation rate were also similar among the comparison groups $(\mathrm{P}=0.91$; $\mathrm{EN}, 36 \pm 2.3 \mathrm{TF}, 35 \pm 1.5$; $\mathrm{UE}, 37 \pm 1.1$; Table 3 ).

\section{Pregnancy outcomes were not affected by endometriosis}

The clinical pregnancy rate in women with endometriosis $(39.7 \%)$ was similar $(P=0.254)$ when compared to those with no endometriosis (TF, 43.6; UE, 38.7\%). We were not able to obtain, however, have the live birth rate.

Embryos from women with endometriosis have longer time to cleave at first cytokinesis but had shorter second cytokinesis

Using Eeva ${ }^{\mathrm{TM}}$ time-lapse system, we found the embryos from women with endometriosis took a longer time to develop $(n=43 ; P<0.0001)$ to the 3-cell stage $(P=273)$ despite a shorter time taken to develop from 2-cell to 4-cell stage $(P<0.0001, n=43)$ when compared to embryos of women without endometriosis.

\section{Discussion}

This study showed that the number of oocytes retrieved per follicle $>14 \mathrm{~mm}$ was lower in women with endometriosis compared to controls, despite similar number of antral follicles and number of mature follicles prior to egg collection. The findings of this study are in line with the results of several metaanalyses (Barnhart et al., 2002, Hamdan et al., 2015a, Hamdan et al., 2015b, Harb et al., 2013). The 
disparity between the number of collected oocytes and the number of mature follicles may be due to the failure of oocytes to mature within the developed follicles, and were therefore not available for retrieval (Hamdan et al., 2016). The oocyte maturation process and follicular growth do not occur simultaneously (Jones and Lane, 2013) and the final maturation for the oocyte usually takes place within 36 following LH surge (natural cycle) or after trigger injection (artificial cycle).

The follicular response to ovarian stimulation appeared to be unaffected by the presence or absence of endometriosis as there were similar number of antral follicle count and mature follicles in all the groups prior to oocyte collection. However, the lower number of oocytes collected suggests the possible impact of the endometriotic disease on oocyte development (Hamdan et al., 2016). There are several factors affecting the physiological oocyte maturation process, for example, intrinsically by high level of oxidative stress, and extrinsically by cigarette smoking, ultraviolet (UV) radiation, or toxic drugs. Despite these effects, a more direct effect to the developing oocyte can be related to an aberrant follicular microenvironment in women with endometriosis. The content of a follicle is physiologically important for oocyte development and in endometriosis, the cellular and molecular milieu from the disease is known to mediate the damage to the ovary and the developing oocytes (Sanchez et al., 2014).

Whilst the fertilisation rate was not statistically different across the groups, the fertilised oocytes in endometriosis group were more likely to arrest and suffer from failure to cleave from fertilisation to the 8cells stage. The higher number of early embryo arrest at D1 suggests the presence of poor oocyte quality (Ajduk et al., 2011).

Despite a higher rate of D1 arrest, embryos of women from endometriosis group that have successfully cleaved were shown in this study to have similar developmental potential. At D3 assessment, the majority of the embryos achieved comparable quality compared to the control groups. The results were unaffected when the graded embryos were re-categorised into 2 groups of good and poor quality embryo. This finding is not unexpected as the poorer quality oocytes, which failed to develop beyond the 8-cell stage, were already excluded. The latter may represent a natural biological triaging mechanism for quality control, so as to prevent poor quality oocytes from developing further, and it therefore follows that the pregnancy rate is not dissimilar when compared to the control groups.

Embryos that were not transferred at day 3 had an extended culture up to day 5, when they were further assessed. At this stage, suitable blastocysts were transferred or cryopreserved for future use. In this study the blastulation rate and FEHB rate were similar in the endometriosis group compared to the control groups, which suggests that the increased aneuploidy rate reported by previous studies may not be apparent at embryological assessment (Vega et al., 2014, Gianaroli et al., 2010). This current study also found similar utilisation rate of embryo between the groups indicated by equal number of embryos transferred and frozen per collected oocytes. This is counter-intuitive as one expects a higher rate of arrest prior to the 8-cell stage in the endometriosis group which in turn leading to a lower number of embryos available for freezing. This study may not be sufficiently large in sample size to delineate the 
difference or that the difference may become more apparent in older women or in women with low ovarian reserve, which are two groups of women not specifically examined in this study.

Although with careful selection of the comparison groups, this study suffers from potential false positive and false negatives in relation to the diagnosis of endometriosis. Participants included in this study were grouped in those with endometriosis and those without. In the endometriosis group, women who had diagnostic laparoscopy did not necessarily received the same surgical treatment (endometriotic spots ablation, excision, or endometrioma cystectomy), it was also not known whether these women had a complete resolution, still have residual disease, or have developed disease recurrence. The progress of the disease following a surgical treatment is unpredictable and may have an impact on the IVF treatment outcomes. The present of heterogeneity of surgical treatmnet and its stages may influence the success rate of IVF treatment (Coccia et al., 2011). However both data were not available. Our inclusion criteria for the control group is likely to have excluded most women with significant endometriosis. The only group of women we were not able to exclude confidently are those with superficial peritoneal endometriosis. However, to include/exclude those with superficial peritoneal endometriosis warrants performing a laparoscopy on every subfertile patient undergoing IVF. The latter is not the current practice, and also not recommended (Dunselman et al., 2014). We therefore do not feel that this is justifiable, especially in the context of a retrospective study.

The mean number of oocytes retrieved and mature oocytes would generally be higher in agonist cycles (Wang et al., 2017, Al-Inany et al., 2016, Xiao et al., 2014) than antagonist and hence, whilst the comparison groups were not balanced in terms of the stimluation regime, with the endometriosis group having a higher number of participants who had long agonist treatment, we believe that this would likely to underestimate the difference rather than exagarate the difference between the two groups.

Embryology data gathered from this study was retrospective and was based on time specific, standardised morphological assessment, which is still subjected to inter- and intra-observer variability. As an alternative, it is now possible to use a non-invasive, time-lapse imaging of the embryos within an undisturbed condition. By using this technology, critical and subtle differences in cell division can be recorded and analysed in greater detail. This will provide more reliable data compared to the former although a larger study on this aspect of the data will in future need to be conducted.

\section{Conclusions}

Our study demonstrated that women with endometriosis have a lower number of oocyte collected per follicle $>14 \mathrm{~mm}$. The fertilisation rate was not dissimilar amongst the comparison groups, but the presence of endometriosis was associated with a higher rate of early embryo arrest, implicating poor oocyte quality. Accepting the limitations discussed, endometriosis did not impact on embryo quality beyond the 8-cell-stage or the overall clinical pregnancy rate.

\section{Abbreviations}


ART Artificial Reproductive Techniques

EN endometriosis

FEHB fully expanded and hatching blastocyst

HPI hours post insemination

ICSI intra-cytoplasmic sperm injection

IVF in-vitro fertilisation (IVF)

TF tubal factor

UE unexplained infertility

\section{Declarations}

\section{Ethics approval and consent to participate:}

Study is approved by University Hospital Southampton NHS Foundation Trust RHM O\&G0213. All procedures performed in studies involving human participants were in accordance with the ethical standards.

\section{Consent for publication:}

Not applicable

\section{Availability of data and material:}

All data generated or analysed during this study are author on reasonable request.

\section{Competing interests}

The authors declare that they have no competing interests.

\section{Funding}

This study was funded by the Complete Fertility Centre Southampton, UK and Ministry of Higher Education Malaysia (sponsor for the first author's PhD). Both funders have no role in the design of the study, data collection, analysis, interpretation of data and in writing the manuscript.

\section{Authors' contribution}

$\mathrm{MH}$ for data analysis and interpretation, manuscript drafting and amendments. 
AP, MC, YC for protocol development, data analysis and interpretation (support), manuscript and drafting and amendments (support). All authors have read and approved the manuscript.

\section{Acknowledgements:}

We thank all our participants. We acknowledge the assistance and cooperation of the embryologists in Complete Fertility Centre Southampton, UK and Wessex Fertility Centre Southampton UK.

\section{Disclosure}

Nothing to disclose for all the authors

\section{References}

1. Ajduk A, llozue T, Windsor S, Yu Y, Seres KB, Bomphrey RJ, Tom BD, Swann K, Thomas A, Graham C, Zernicka-Goetz M. Rhythmic Actomyosin-Driven Contractions Induced By Sperm Entry Predict Mammalian Embryo Viability. Nat Commun. 2011;2:417.

2. Al-Fadhli R, Kelly SM, Tulandi T, Tanr SL. Effects Of Different Stages Of Endometriosis On The Outcome Of In Vitro Fertilization. J Obstet Gynaecol Can. 2006;28:888-91.

3. Al-Inany HG, Youssef MA, Ayeleke RO, Brown J, Lam WS, Broekmans FJ. Gonadotrophin-Releasing Hormone Antagonists For Assisted Reproductive Technology. Cochrane Database Syst Rev. 2016;4:Cd001750.

4. Alpha S In Reproductive, M. \& Embryology, E. S. I. G. O. 2011. The Istanbul Consensus Workshop On Embryo Assessment: Proceedings Of An Expert Meeting. Hum Reprod, 26, 1270-83.

5. Barnhart K, Dunsmoor-Su R, Coutifaris C. Effect Of Endometriosis On In Vitro Fertilization. Fertil Steril. 2002;77:1148-55.

6. Coccia ME, Rizzello F, Mariani G, Bulletti C, Palagiano A, Scarselli G. Impact Of Endometriosis On In Vitro Fertilization And Embryo Transfer Cycles In Young Women: A Stage-Dependent Interference. Acta Obstet Gynecol Scand. 2011;90:1232-8.

7. Da Broi MG, Malvezzi H, Paz CC, Ferriani RA, Navarro PA. Follicular Fluid From Infertile Women With Mild Endometriosis May Compromise The Meiotic Spindles Of Bovine Metaphase li Oocytes. Hum Reprod. 2014;29:315-23.

8. Diaz I, Navarro J, Blasco L, Simon C, Pellicer A, Remohi J. Impact Of Stage lii-Iv Endometriosis On Recipients Of Sibling Oocytes: Matched Case-Control Study. Fertil Steril. 2000;74:31-4.

9. Dong X, Liao X, Wang R, Zhang H. The Impact Of Endometriosis On Ivf/Icsi Outcomes. Int J Clin Exp Pathol. 2013;6:1911-8.

10. Dunselman GA, Vermeulen N, Becker C, Calhaz-Jorge C, D'hooghe T, De Bie B, Heikinheimo O, Horne AW, Kiesel L, Nap A, Prentice A, Saridogan E, Soriano D, Nelen W. European Society Of Human, R. \& 
Embryology 2014. Eshre Guideline: Management Of Women With Endometriosis. Hum Reprod, 29, 400-12.

11. Filippi F, Benaglia L, Paffoni A, Restelli L, Vercellini P, Somigliana E, Fedele L. 2014. Ovarian Endometriomas And Oocyte Quality: Insights From In Vitro Fertilization Cycles. Fertil Steril, 101, 988 - 93 E1.

12. Gianaroli L, Magli MC, Cavallini G, Crippa A, Capoti A, Resta S, Robles F, Ferraretti AP. Predicting Aneuploidy In Human Oocytes: Key Factors Which Affect The Meiotic Process. Hum Reprod. 2010;25:2374-86.

13. Hamdan M, Dunselman G, Li TC, Cheong Y. The Impact Of Endometrioma On Ivf/Icsi Outcomes: A Systematic Review And Meta-Analysis. Hum Reprod Update. 2015a;21:809-25.

14. Hamdan M, Jones KT, Cheong Y, Lane SI. The Sensitivity Of The Dna Damage Checkpoint Prevents Oocyte Maturation In Endometriosis. Sci Rep. 2016;6:36994.

15. Hamdan M, Omar SZ, Dunselman G, Cheong Y. Influence Of Endometriosis On Assisted Reproductive Technology Outcomes: A Systematic Review And Meta-Analysis. Obstet Gynecol. 2015b;125:79-88.

16. Harb HM, Gallos ID, Chu J, Harb M, Coomarasamy A. The Effect Of Endometriosis On In Vitro Fertilisation Outcome: A Systematic Review And Meta-Analysis. Bjog. 2013;120:1308-20.

17. Jones KT, Lane SI. Molecular Causes Of Aneuploidy In Mammalian Eggs. Development. 2013;140:3719-30.

18. Lin XN, Wei ML, Tong XM, Xu WH, Zhou F, Huang QX, Wen GF, Zhang SY. Outcome Of In Vitro Fertilization In Endometriosis-Associated Infertility: A 5-Year Database Cohort Study. Chin Med J (Engl). 2012;125:2688-93.

19. Mansour G, Aziz N, Sharma R, Falcone T, Goldberg J, Agarwal A. The Impact Of Peritoneal Fluid From Healthy Women And From Women With Endometriosis On Sperm Dna And Its Relationship To The Sperm Deformity Index. Fertil Steril. 2009;92:61-7.

20. Mansour G, Sharma RK, Agarwal A, Falcone T. Endometriosis-Induced Alterations In Mouse Metaphase li Oocyte Microtubules And Chromosomal Alignment: A Possible Cause Of Infertility. Fertil Steril. 2010;94:1894-9.

21. Mekaru K, Yagi C, Asato K, Masamoto H, Sakumoto K, Aoki Y. Effects Of Early Endometriosis On IvfEt Outcomes. Front Biosci (Elite Ed). 2013;5:720-4.

22. Munne S, Chen S, Colls P, Garrisi J, Zheng X, Cekleniak N, Lenzi M, Hughes P, Fischer J, Garrisi M, Tomkin G, Cohen J. Maternal Age, Morphology, Development And Chromosome Abnormalities In Over 6000 Cleavage-Stage Embryos. Reprod Biomed Online. 2007;14:628-34.

23. Rajani S, Chattopadhyay R, Goswami SK, Ghosh S, Sharma S, Chakravarty B. Assessment Of Oocyte Quality In Polycystic Ovarian Syndrome And Endometriosis By Spindle Imaging And Reactive Oxygen Species Levels In Follicular Fluid And Its Relationship With Ivf-Et Outcome. J Hum Reprod Sci. 2012;5:187-93.

24. Reinblatt SL, Ishai L, Shehata F, Son WY, Tulandi T, Almog B. a. Effects Of Ovarian Endometrioma On Embryo Quality. Fertility Sterility. 2011;95:2700-2. 
25. Reinblatt SL, Ishai L, Shehata F, Son WY, Tulandi T, Almog B. b. Effects Of Ovarian Endometrioma On Embryo Quality. Fertil Steril. 2011;95:2700-2.

26. Sanchez AM, Vigano P, Somigliana E, Panina-Bordignon P, Vercellini P, Candiani M. The Distinguishing Cellular And Molecular Features Of The Endometriotic Ovarian Cyst: From Pathophysiology To The Potential Endometrioma-Mediated Damage To The Ovary. Hum Reprod Update. 2014;20:217-30.

27. Senapati S, Sammel MD, Morse C, Barnhart KT. Impact Of Endometriosis On In Vitro Fertilization Outcomes: An Evaluation Of The Society For Assisted Reproductive Technologies Database. Fertil Steril. 2016;106:164-71 E1.

28. Simon C, Gutierrez A, Vidal A, De Los Santos MJ, Tarin JJ, Remohi J, Pellicer A. Outcome Of Patients With Endometriosis In Assisted Reproduction: Results From In-Vitro Fertilization And Oocyte Donation. Hum Reprod. 1994;9:725-9.

29. Suzuki T, Izumi S, Matsubayashi H, Awaji H, Yoshikata K, Makino T. Impact Of Ovarian Endometrioma On Oocytes And Pregnancy Outcome In In Vitro Fertilization. Fertility Sterility. 2005;83:908-13.

30. Vega M, Breborowicz A, Moshier EL, Mcgovern PG, Keltz MD. Blastulation Rates Decline In A Linear Fashion From Euploid To Aneuploid Embryos With Single Versus Multiple Chromosomal Errors. Fertil Steril. 2014;102:394-8.

31. Wang R, Lin S, Wang Y, Qian W, Zhou L. Comparisons Of Gnrh Antagonist Protocol Versus Gnrh Agonist Long Protocol In Patients With Normal Ovarian Reserve: A Systematic Review And MetaAnalysis. Plos One. 2017;12:E0175985.

32. Xiao JS, Su CM, Zeng XT. Comparisons Of Gnrh Antagonist Versus Gnrh Agonist Protocol In Supposed Normal Ovarian Responders Undergoing Ivf: A Systematic Review And Meta-Analysis. Plos One. 2014;9:E106854.

33. Xu B, Guo N, Zhang XM, Shi W, Tong XH, Iqbal F, Liu YS. Oocyte Quality Is Decreased In Women With Minimal Or Mild Endometriosis. Sci Rep. 2015;5:10779.

\section{Tables}

Due to technical limitations, Tables 1-4 are only available as a download in the supplemental files section

\section{Supplementary Files}

This is a list of supplementary files associated with this preprint. Click to download.

- Table3.jpg

- Table1.jpg

- Table2.jpg

- Table4.jpg 